\title{
LINC00152 Acts as a Potential Marker in Gliomas and Promotes Tumor Proliferation and Invasion Through The LINC00152/miR-107/RAB10 Axis
}

qing liu ( $\square$ liuqingdr@csu.edu.cn )

Xiangya Hospital Central South University https://orcid.org/0000-0003-1357-4866

gang peng

Xiangya Hospital Central South University

Jun Su

Xiangya Hospital Central South University

zeyou wang

Second Xiangya Hospital

songhua xiao

Sun Yat-Sen University

\section{Research Article}

Keywords: long non-coding RNA, LINC00152/MIR-107/RAB10 axis, glioblastoma, proliferation, invasion, prognosis

Posted Date: June 22nd, 2021

DOl: https://doi.org/10.21203/rs.3.rs-615132/v1

License: (9) (1) This work is licensed under a Creative Commons Attribution 4.0 International License. Read Full License 


\section{Abstract}

Aberrant expression of long noncoding RNAs plays a pivotal role in tumorigenesis. Recently, several studies have showed that the LINC00152 gene is upregulated in a variety of tumors and plays an oncogene role; however, its underlying molecular mechanisms in glioblastoma remain unclear. In this study, we found that LINC00152 was upregulated in gliomas and its expression was significantly associated with high tumor aggressiveness and poor outcomes for glioma patients through bioinformatics analysis. Functionally, the knockdown of LINC00152 not only inhibited malignant behaviors of glioma, such as proliferation and invasion of glioma cells and induced apoptosis in vitro but also suppressed tumorigenesis in vivo. Mechanistically, results of the bioinformatics analysis and experimental studies confirmed that $L I N C 00152$ and RAB10 as the targets of miR-107, and LINC00152 might act as a sponge for $m i R-107$ to regulate the expression of $R A B 10$ in glioblastoma. Additionally, silencing miR-107 reversed the effects induced by LINC00152 knockdown on glioblastoma cells both in vitro and in vivo. Taken together, our data suggested that $L I N C 00152$ is a candidate prognostic marker of glioma, and that the LINC00152/MIR-107/RAB10 axis plays a pivotal role in regulation of the glioma malignancy, and therefore, targeting the axis might be an effective therapeutic strategy to treat glioma.

\section{Introduction}

Glioma, the most common and aggressive primary brain tumor arising from glial cells in the central nervous system, is highly invasive and resistant to traditional therapies. It accounts for approximately $80 \%$ of primary malignant brain tumors(Ricard et al. 2012; Lathia et al. 2015;Wang et al. 2015;Siegel et al.

2017). Glioblastoma multiforme (GBM) is the most frequent and severe subtype of glioma accounting for $50 \%$ of diffuse gliomas(Furnari et al. 2015; Lathia et al. 2015; Yuan et al. 2015). Despite multimodal treatments, including surgery, radiotherapy, and chemotherapy, which are constantly being improved, the average survival time of GBM patients is only approximately 15 months from diagnosis(Omuro and DeAngelis 2013). Therefore, it is necessary to completely understand the molecular mechanisms underlying GBM invasion and metastasis and to develop novel therapeutic strategies.

Long non-coding RNAs (LncRNAs) are a group of endogenous small RNAs of over 200 nucleotides long that do not have a protein-coding capacity(Huarte 2015;Beermann et al. 2016;Deguchi et al. 2017;Fan et al. 2017;Gomes et al. 2017). Over the past decade, IncRNAs have been described in a variety of processes including evolution(Gummalla et al. 2014), embryonic development(Floris et al. 2017), metabolism(Huarte 2015;Beermann et al. 2016), and oncogenesis(Fang et al. 2015;Gomes et al. 2017). Mechanistically, IncRNAs with aberrant expression levels play critical roles at the transcriptional and posttranscriptional levels in cancer biology(Fang et al. 2015;Huarte 2015;Beermann et al. 2016). Recently, many IncRNAs have been reported to be aberrantly expressed and involved in tumor initiation and progression of glioma. For example, miR-155 host gene ( $m i R 155 H G)$ is highly expressed in glioblastoma cells and facilitates glioma progression(Wu et al. 2017). HOTAIR, a cell cycle-associated IncRNA, is preferentially expressed in classical and mesenchymal glioma cells and is involved in the regulation of cell proliferation in glioblastoma(Zhang et al. 2013;Pastori et al. 2015). Other IncRNAs, such as 
CCAT2(Guo et al. 2016), HULC(Zhu et al. 2016), and H19(Jia et al. 2016) regulate angiogenesis and behavior of glioma cells. TUG1 promotes glioma stem cell (GSC) self-renewal and growth and inhibit GSC differentiation(Katsushima et al. 2016). In addition, these IncRNAs are potential therapeutic targets for glioblastoma cells. HOTAIR regulates the permeability of blood-tumor barrier (BTB) via binding to miR148b-3p, which further targets USF1 in glioma microvascular endothelial cells, thus, HOTAIR/miR-148b$3 p /$ USF 1 axis is a potential novel drug target for glioma treatment(Sa et al. 2017). XIST IncRNA increases BTB permeability and inhibits glioma angiogenesis by targeting miR-137 and might be a potential therapeutic target in glioma samples(Yu et al. 2017).

Long intergenic non-coding RNA 152 (LINC00152) is 828 nucleotides long and is located on chromosome $2 \mathrm{p} 11.2$ in an intergenic region between the pseudogenes platelet-activating factor acetylhydrolase $1 \mathrm{~b}$ regulatory subunit 1 pseudogene 1 (PAFAH1B1P1) and LOC107985796(Teng et al. 2017;Yu et al. 2017). During the past few years, accumulating evidence have indicated that $L I N C 00152$ is upregulated and plays an oncogene role in several carcinomas, including promoting cell proliferation and metastasis and inhibiting apoptosis(Ji et al. 2015;Chen et al. 2017;Feng et al. 2017). Besides, LINC00152 might also act as a diagnostic and prognostic biomarker for different cancers(Chen et al. 2017;Feng et al. 2017). The abnormal expression of LINC00152 in glioma tissues and GSCs has been reported(Yu et al. 2017). However, the biological role and underlying molecular mechanisms of LINC00152 in glioblastoma cells is still unclear.

In this study, we combined bioinformatics analysis and experimental studies to investigate the expression pattern of LINC00152, its biological function, and the underlying mechanism in gliomas.

\section{Materials And Methods}

\section{Cell lines and cultures}

Human glioma cell lines (U251 and U87) were obtained from the American Type Culture Collection (Manassas, VA, USA). All cell lines were subjected to a short tandem repeat test before this study. Every cell line was passaged less than 10 times during the experiments. Individual cell lines were maintained according to the supplier's instructions. Cells were cultured in medium supplemented with $10 \%$ fetal bovine serum and antibiotics (100 units $/ \mathrm{mL}$ penicillin and $100 \mu \mathrm{g} / \mathrm{mL}$ streptomycin) and were incubated at $37^{\circ} \mathrm{C}$ in a humidified incubator with $5 \% \mathrm{CO}_{2}$.

\section{Human tissue samples}

Patients with glioma who were newly diagnosed, treated, and followed at the Department of Neurosurgery, Xiangya Hospital, Central South University, Hunan, China were enrolled for this study. We obtained frozen tissue samples from 73 gliomas and 78 normal brain tissues between March 2008 and November 2010. This study was approved by the hospital institutional review board and written informed consent was obtained from all patients. All the protocols were reviewed by the Joint Ethics Committee of the Central South University Health Authority and were performed following national guidelines. Tissue 
samples were collected during surgery and diagnosed using the World Health Organization (WHO) criteria by two pathologists who were blinded to patient data. Tissues were frozen in RNAlater (Ambion) in liquid nitrogen and stored until total RNA or protein were extracted. Clinical data, including gender, age, followup, and outcome, were obtained from medical records.

\section{Cells transfection}

Cell transfection was performed using Lipofectamine 2000 (Invitrogen-Life Technologies, Carlsbad, CA, USA) as per the manufacturer's instructions. Cells were seeded in cell culture dishes or plates and were grown overnight. On the following day, the cells were transfected with miRNA mimics/NC using Lipofectamine 2000 and incubated. After $72 \mathrm{~h}$ incubation, these cells were subjected to western blot analysis and also other assays.

\section{Vector construction}

The pLKO.1-puro vector used for the stable expression of shRNA against LINC00152 (sh-linc) contained a puromycin resistance gene. The scrambled control shRNA (sh-ctr) sequence had no homology to any human genomic sequences. The cultured cells $\left(3 \times 10^{5}\right.$ cells/well) were seeded in 6-well culture plates and maintained in DMEM medium containing $10 \%$ FBS for $24 \mathrm{~h}$ before transfection. Cell transfection was performed using Lipofectamine 2000 (Invitrogen-Life Technologies, Carlsbad, CA, USA) as per the manufacturer's instructions. For screening, puromycin $(1 \mu \mathrm{g} / \mathrm{mL})$ was added to the medium $72 \mathrm{~h}$ after transfection. The medium was replaced every $2 \mathrm{~d}$ for 2-3 weeks. U251 and U87 cells with high endogenous LINC00152 expression were selected for silencing. The expression levels of LINC00152 was confirmed by qRT-PCR.

\section{Quantitative real-time PCR (qRT-PCR) assays}

RNA was isolated from harvested cells or human tissues with Trizol reagent according to the manufacturer's instructions (Invitrogen, CA, USA). One $\mu \mathrm{g}$ of total RNA was reverse transcribed to cDNA using a Reverse Transcription Kit (Thermo Fisher Scientific, MA, USA). qRT-PCR was performed using SYBR Premix DimerEraser kit (Takara, Dalian, China) on a CFX96 Real-Time PCR Detection System (BioRad, CA, USA) to determine the relative expression levels of target genes. Expression of each gene was quantified by measuring Ct values and normalized using the $2^{-\Delta \Delta c t}$ method. U6 small nuclear (snRNA) and GAPDH mRNA were used as an internal control for mRNA and mature miRNA, respectively. The primers used were showed as follows: LINC00152: F: 5'-AAAATCACGACTCAGCCCCC-3' and R: 5'AATGGGAAACCGACCAGACC-3'; miR-107: F: 5'-GGAGCAGCATTGTACAGG-3' and R: 5'CAGTGCGTGTCGTGGA-3'; RAB10: F: 5'-TTTCACACCATCACAACCTCC-3' and R: 5'GGTACAACTCTTTTTGTCGTCC-3'; U6: F: 5'-CTCGCTTCGGCAGCACA-3' and R: 5'AACGCTTCACGAATTTGCGT-3; and GAPDH: F: 5'-GGGAGCCAAAAGGGTCAT -3' and R: 5'GTCCTTCCACGATACCAA-3'.

\section{Cell Counting kit-8 assay}


Cell proliferation of GBM cells was measured by Cell Counting Kit-8 (CCK-8; Sigma-Aldrich, Shanghai, China) according to the manufacturer's instructions. Briefly, U251 and U87 cells transfected with sh-linc or sh-ctr were seeded at a density of $2 \times 10^{3}$ cells per well in 96 -well plates. The cells were incubated for 24 , 48,72 , and $96 \mathrm{~h}$ after transfection. At each of the desired time points, CCK-8 solution was added (10 $\mu \mathrm{L} /$ well) to the cells and incubated for $2 \mathrm{~h}$ at $37^{\circ} \mathrm{C}$, followed by absorbance measurements at $420 \mathrm{~nm}$ using a microplate reader (Model 680 microplate reader, Bio-Rad Laboratories). Each assay was performed in five replicates.

\section{Colony formation assay}

After transfection, the cells were digested using $0.25 \%$ trypsin, and then cells were suspended in medium for counting. Cells were seeded onto a 6 -well plate at $1 \times 10^{3}$ cells per well and were incubated at $37^{\circ} \mathrm{C}$ with $5 \% \mathrm{CO}_{2}$ containing saturated humidity for $14 \mathrm{~d}$ and the growth medium was replaced once in three days. After clone formation, the supernatant was discarded, and the plate was carefully immersed twice with PBS. The cells were fixed by adding $4 \%$ paraformaldehyde and incubation for $15 \mathrm{~min}$. Then, the fixing solution was removed and appropriate amounts of Crystal Violet Staining Solution was added and incubated for 30 min and then the staining solution was slowly washed away using running water. After air drying, the resulting colonies were then counted.

\section{Flow cytometric analysis of apoptosis}

Cellular apoptosis was assessed by Annexin V/propidium iodide (PI) staining and flow cytometry was performed using an Annexin V-fluorescein Isothiocyanate Apoptosis Detection Kit (Beyotime Biotechnology, Shanghai, China) according to the manufacturer's instructions. Briefly, the cells were digested by trypsin, centrifuged, and washed twice with phosphate buffered saline (PBS). Then the harvested cells $\left(5^{\prime} 10^{5}\right)$ were resuspended in Annexin $V$ binding buffer. After staining with Annexin $V$ and $\mathrm{PI}$, the cells were analyzed with the help of a flow cytometer. All experiments were repeated at least three times.

\section{Cell Matrigel invasion assay}

Matrigel invasion migration was evaluated using a Transwell migration assay. Briefly, filters coated with Matrigel in the upper compartment were loaded with $200 \mu \mathrm{L}$ of serum-free medium containing $5 \times 10^{4}$ transfected cells, and the lower compartment was filled with $20 \% \mathrm{FBS}$. After $24 \mathrm{~h}$, cells migrated to the bottom surface were fixed with $100 \%$ methanol and were counted after staining with $0.5 \%$ crystal violet. The number of invaded cells were counted in six randomly selected fields under a microscope and the average value was calculated. Each experiment was conducted in triplicate.

\section{Western blot analysis}

Western blot was performed as described previously(Wang et al. 2016). Briefly, cells were lysed and the total proteins were extracted and quantified. Equivalent amounts of protein from each sample were 
separated by sodium dodecyl sulphate-polyacrylamide gel electrophoresis (SDS-PAGE) and transferred to polyvinylidene fluoride (PVDF) membranes (Millipore, USA). The membrane was blocked with $5 \%$ skim milk at room temperature for $1 \mathrm{~h}$, followed by incubation with primary antibody (RAB10 with 1:1000 dilution, GAPDH with 1:5000 dilution) at $4{ }^{\circ} \mathrm{C}$ overnight and detected by chemiluminescence. Antibodies against RAB10 (\#4262) were obtained from Cell Signaling Technology (Beverly, MA, USA) and antibodies against GAPDH (sc-32233) were obtained from Santa Cruz Biotechnology (Santa Cruz, CA, USA).

\section{Luciferase reporter assays}

Luciferase reporter assays were performed as described previously(Peng et al. 2015). Briefly, the luciferase reporter plasmids containing wild-type (wt) or mutant (mut) LINC00152 genes were purchased from GeneChem (Shanghai, China). phRL-TK plasmid was used as an internal control. Glioma cells (2 $\times 10^{5}$ ) were plated in 24-well plates, and then cotransfected with either wildtype or mut $L$ INCO0152 luciferase reporter plasmid, together with miR-107 mimics or miR-NC. After incubation for $24 \mathrm{~h}$, luciferase activities were measured using a Dual-Luciferase Reporter Assay System kit (Promega) according to the manufacturer's instructions.

\section{Wound healing assay}

Glioma cells were cultured in 6-well plates until 80\% confluency was reached. Subsequently, cells were then transfected with LINC00152 shRNA or control shRNA. Cultures were scratched using a $10 \mathrm{Ml}$ tip 6-h post transfection to form wound gaps. The wound gaps were photographed 12, 24, and $36 \mathrm{~h}$ following the scratch. Fields containing wounds were visualized and the distance migrated by cells was measured from five different areas for each wound.

\section{Mouse xenograft models}

All the animal procedures were performed in accordance with institutional guidelines. Ethical approval was obtained from the Institute Research Ethics Committee of Central South University. For subcutaneous implantation, U251 cells stably transfected with sh-linc or sh-ctr were collected and suspended in PBS buffer at a concentration of $1 \times 10^{7}$ cells $/ \mathrm{ml}$. Tumor growth was monitored by caliper measurement once or twice a week. After $35 \mathrm{~d}$, mice were euthanized, and tumors were extracted for immunohistochemical analysis of Ki-67.

To assay the effects of LINC00152 on tumor formation after miR-107 knockdown, U251 cells stably transfected with sh-linc (10 mice) or sh-ctr (5 mice) were collected and suspended in PBS buffer at a concentration of $1 \times 10^{7}$ cells $/ \mathrm{ml}$. Aliquots of $40 \mu \mathrm{lPBS}$ containing $1 \mu \mathrm{g}$ of miR-NC were directly injected into the tumors of the sh-ctr group mice. The sh-linc group mice were divided into two groups. Aliquots containing $40 \mu \mathrm{l}$ PBS with $1 \mu \mathrm{g}$ of miR-107inhibitors or miR-NC were directly injected into the tumor. Tumor growth was monitored by caliper measurement once or twice a week for at least 5 weeks. After 35 d, mice were euthanized, and tumors extracted. 


\section{Bioinformatics analysis}

Three publicly available databases, including The TCGA lower grade glioma and glioblastoma (GBMLGG) dataset (https://xenabrowser.net/), the Gene Expression Omnibus (GEO) GSE16011 (https://hgserver1.amc.nl/cgi-bin/r2/main.cgi) and CGGA dataset (http://www.cgga.org.cn/), were used in this study. Gene expression analysis and Survival analysis were performed, and the detail of them was described in previous publications(Su et al. 2019;Su et al. 2019).

\section{Statistical analysis}

All experiments were performed three times and data were analyzed using GraphPad Prism 5 (La Jolla, CA, USA). Statistical differences between groups were analyzed using Student's t-test, one-way ANOVA, and $\chi^{2}$ tests, using the SPSS 17.0 program and RStudio. A $p$-value of $<0.05$ was considered to indicate a statistically significant result.

\section{Results}

\section{LINC00152 is upregulated in glioma tissues and its expression is associated with glioma patient outcomes}

LINC00152 is an oncogene that promotes cell proliferation, invasion, and migration in various tumors(Yu et al. 2017). To investigate the expression of LINC00152 in gliomas and normal brain tissues, the GSE16011 database was used. The result showed that the expression of LINC00152 was significantly upregulated in gliomas compared to levels in normal tissue ( $p<0.05$, Fig. 1a). Furthermore, we analyzed the expression of LINC00152 in different grades of glioma and found that its level increases with WHO grades based on the GSE16011 database (Fig. 1a), which was well validated in TCGA and CGGA datasets (Fig. 1b and supplementary Fig. 1a). Moreover, LINC00152 expression was significantly correlated with MGMT promoter status and IDH status of glioma. LINC00152 expression in IDH-Wt gliomas was markedly higher than that in IDH-Mut gliomas based on TCGA database (Fig. 2c), consistent with result obtained from the CGGA database (Supplementary Fig. 1b). LINC00152 expression in gliomas with MGMT promoter unmethylation was significantly higher than that in gliomas with MGMT promoter methylation based on TCGA database (Fig. 2d). In addition, LINC00152 expression was significantly upregulated in the mesenchymal and classical subtype compared with other two respective molecular subtypes in the TCGA dataset (Fig. 1e).

Since LINC00152 expression significantly associated with WHO grade and molecular subtype in gliomas, we further investigated its prognostic value and the Kaplan-Meier survival analysis was performed.

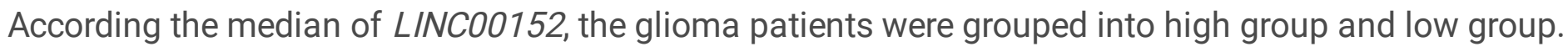
Based on the TCGA database, the result indicated that high expression of LINC00152 is significantly associated with poor prognosis in glioma patients (Fig. 1f), which was well validated in CGGA dataset (supplementary Fig. 1c). To investigate whether LINC00152 could be an independent prognostic marker for glioma, we simultaneously performed univariate and multivariate Cox regression analysis based on 
the TCGA dataset. Univariate Cox analysis showed that LINC00152 expression, patient age at diagnosis, WHO grade, MGMT promoter status, IDH status and transcriptome subtype were significantly associated with overall survival of glioma patients. According to multivariate Cox analysis, the LINC00152 expression was still a significant predictive factor after adjusting for the aforementioned clinical factors (Table 1).

Table 1

Univariate and multivariable Cox regression analysis of overall survival based on TCGA dataset

\begin{tabular}{|c|c|c|c|c|}
\hline & \multicolumn{2}{|c|}{ Univariate analysis } & \multicolumn{2}{|l|}{ Multivariate analysis } \\
\hline & $\begin{array}{l}\text { HR } \\
(95 \% \text { Cl for HR) }\end{array}$ & $\mathrm{P}$ & $\begin{array}{l}\text { HR } \\
(95 \% \mathrm{Cl} \text { for HR) }\end{array}$ & $P$ \\
\hline LINC00152 & $\begin{array}{l}1.645(1.551- \\
1.745)\end{array}$ & 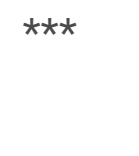 & $\begin{array}{l}1.206 \\
(1.081-1.345)\end{array}$ & $\star \star \star *$ \\
\hline $\begin{array}{l}\text { gender } \\
\text { male VS Female }\end{array}$ & $\begin{array}{l}1.225(0.955- \\
1.572)\end{array}$ & 0.110 & - & - \\
\hline $\begin{array}{l}\text { Age } \\
\leq 40 \text { VS }>40\end{array}$ & $\begin{array}{l}0.231(0.165- \\
0.324)\end{array}$ & $\star \star \star *$ & $\begin{array}{l}0.3947(0.2565- \\
0.6074)\end{array}$ & 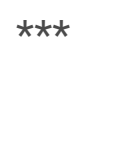 \\
\hline $\begin{array}{l}\text { grade } \\
\text { WHO II VS WHO III + IV }\end{array}$ & $\begin{array}{l}0.178(0.121- \\
0.262)\end{array}$ & 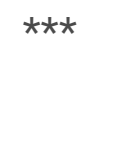 & $\begin{array}{l}0.4497(0.2768- \\
0.7306)\end{array}$ & $\star \star \star$ \\
\hline $\begin{array}{l}\text { IDH status } \\
\text { WT VS MT }\end{array}$ & $\begin{array}{l}10.050 \\
(7.524-13.43)\end{array}$ & $\star \star \star$ & $3.205(1.863-5.514)$ & $\star \star \star *$ \\
\hline $\begin{array}{l}\text { MGMT promoter status } \\
\text { Methylation VS } \\
\text { Unmethylation }\end{array}$ & $\begin{array}{l}3.228 \text { (2.447- } \\
4.259)\end{array}$ & $\star \star \star$ & - & - \\
\hline $\begin{array}{l}\text { Transcriptome subtype } \\
\text { NE + PN VS CL + ME }\end{array}$ & $\begin{array}{l}0.152(0.113- \\
0.203)\end{array}$ & 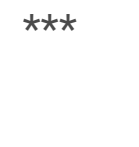 & $1.012(0.6335-1.618)$ & 0.959 \\
\hline
\end{tabular}

Taken together, these results indicated that the expression of LINC00152 was significantly upregulated in GBM and significantly correlated with WHO grade, IDH status, MGMT promoter status and transcriptome subtype of gliomas. Importantly, LINC00152 is an independent prognostic marker for glioma patients and high expression indicates poor clinical prognosis.

The role of LINC00152 in glioma cell proliferation, cell migration, and cell apoptosis 
Cell proliferation and invasion are significantly processed in cancer progression(Fang et al. 2015; Huarte 2015; Lathia et al. 2015). To explore the functions of LINC00152 in glioma, we used a short hairpin RNA (shRNA) to decrease the expression levels of LINC00152 in U251 and U87 cells. qRT-PCR analysis showed that this shRNA can downregulate the expression of LINC00152 in both the cell lines with high efficiency (Fig. 2a). As a functional assay, CCK-8 assay was performed to monitor the effects of LINC00152 on cell proliferation and the results showed that knockdown of LINC00152 expression in U251 and U87 cells caused significant inhibition on cell proliferation (Fig. 2b). On the other hand, LINC00152 knockdown can significantly diminish colony formation and invasion abilities of U251 and U87 cells (Fig. 2c and 2d). Furthermore, flow cytometric analysis demonstrated that LINC00152 silencing in U251 and U87 cells induced cell apoptosis and the percentage of apoptotic cells was significantly increased in the LINC00152 knockdown group when compared to that in the control group which were transfected with control shRNA (Fig. 2e). Taken together, these results suggest that LINC00152 plays an important role in the regulation of cell proliferation, invasion, and apoptosis in glioma cells.

\section{LINC00152 regulates tumor growth in vivo}

To confirm the functional effects of LINC00152 on glioma cells in vivo, we subcutaneously injected U251 cells with stable LINC00152 knockdowns, or U251 cells were transfected with sh-ctr into the right flanks of 4-6 week-old nude mice. After $35 \mathrm{~d}$, tumors were visible and all the mice were euthanized to harvest xenografts. There was a significant reduction in tumor growth in xenografts with LINC00152 knockdown compared to negative controls and the relative tumor weight in the LINC00152 knockdown group was significantly lighter than that in the control group (Fig. 3a and 3b). H\&E staining showed that the tumor tissues were isolated from xenograft mice model (Fig. 3c). In addition, immunohistochemistry (IHC) analysis revealed that the expression of Ki-67 in LINC00152 knockdown group was lower than that in the control group (Fig. 3d). Taken together, these data verify that LINC00152 promotes the growth of GBM cells in vivo.

\section{LINC00152 is a target of miR-107}

LncRNAs are known to act as 'sponges' to sequester endogenous miRNAs to regulate miRNA targets. Therefore, StarBase v2.0 software (http://starbase.sysu.edu.cn/index.php) was used to identify all potential miRNAs that can bind to $L I N C 00152$ gene and the software returned 6 hits, including miR-107, miR-376c-3p, miR-193b-3p, miR-193a-3p, miR-103a-3p, and miR-155-5p. Besides, it has been previously reported that miR-107 is downregulated in glioma and overexpression of miR-107could inhibit proliferation of glioma(Chen et al. 2016), which compelled us to investigate whether LINC00152 was a real target of miR-107. The site on LINC00152 that miR-107 could potentially bind is shown in Fig. $4 \mathrm{a}$. We also analyzed the expression levels of miR-107 in glioma and normal tissues and further investigated the correction factors between LINC00152 expression and miR-107 expression. The result showed that the expression of miR-107 negatively correlated with the expression of $L I N C 00152(\mathrm{r}=-0.447, \mathrm{p}<0.05$, Fig. 4b). Luciferase reporter assays showed that miR-107 mimics significantly suppressed luciferase activity in U251 and U87 cells that carried plasmids with wildtype rather than mutant 3'-UTR of 
LINC00152 (Fig. 4C), which revealed that LINC00152 was a direct target of miR-107. In addition, LINC00152 silencing could upregulate miR-107 expression in both U251 and U87 cells (Fig. 4d). Taken together, these findings demonstrated that LINC00152 was a direct target of miR-107 and LINC00152 could suppress expression levels of miR-107 in glioma cells.

\section{RAB10 is a target of miR-107}

Next, we aimed at identifying the target genes of miR-107via TargetScan (http://www. targetscan.org/), and observed that $R A B 10$ was predicted as a potential target of miR-107. RAB10, a member of the Ras small GTPase superfamily, is a protein-coding gene with GTP and GDP binding domains. Indeed, recent studies demonstrated that $R A B 10$ is closely associated with the genesis and development of certain cancers(Jiang et al. 2016;Wang et al. 2017). Thus, dual luciferase reporter assays were performed to confirm whether miR-107 binds to the putative miR-107 binding site at the 3 '-UTR of RAB10 (Fig. 5a). The results showed that luciferase intensity was significantly attenuated by co-transfected miR-107 mimics and $R A B 10$-WT vectors, but not in the mutant vector lacking the putative miR-107 binding site in both U251 and U87 cells. Also, miR-NC also could not affect the luciferase intensity of RAB10-WT/MT vectors (Fig. $5 \mathrm{~b}$ ). These results suggested that $R A B 10$ is a direct target of miR-107. Then, qRT-PCR and western blotting were performed to assess whether miR-107 could negatively regulate the expression of $R A B 10$ at both the mRNA and protein levels in GBM cell lines, respectively. As expected, miR-107 mimics decreased $R A B 10$ mRNA levels, and conversely, miR-107 inhibitor increased RAB10 mRNA levels (Fig. 5c). Similar results were observed by using western blot analysis (Fig. $5 \mathrm{~d}$ ). These results indicated that $R A B 10$ is a direct binding target of $m i R-107$ and its expression can be regulated by miR-107 levels in glioma samples.

\section{- LINC00152 regulates the expression of RAB10 depending on miR-107}

Since, LINC00152 harbors an identical miR-107 binding site as RAB10, we wanted to know whether $L I N C 00152$ might regulate the expression of $R A B 10$ through miR-107. Firstly, the expression of $L I N C 00152$ and $R A B 10$ in glioma tissues were measured and correlation analysis was performed to detect the potential association between them. The results showed that the expression of $R A B 10$ was not only upregulated in glioma tissues, but also had significantly positive correlation with the expression of LINC00152 (Fig. 6a and 6b). Furthermore, knockdown of LINC00152, in U251 and U87 cell lines, decreased the mRNA levels of $R A B 10$ (Fig. $6 \mathrm{c}$ ) and diminished the expression of $R A B 10$ at the protein level (Fig. 6d). However, overexpression of miR-107 attenuated the effect of LINC00152 silencing on the regulation of $R A B 10$ expression (Fig. $6 \mathrm{c}$ and $6 \mathrm{~d}$ ). In summary, these results indicate that $L I N C 00152 \mathrm{can}$ regulate expression of $R A B 10$ depending on miR-107 in GBM cells.

\section{- miR-107 reverses the functions of LINC00152 in glioma}

To further validate the interactions between LINC00152 and miR-107, we investigated whether miR-107 silencing can rescue the effects caused by LINC00152 knockdown, including cell proliferation, clone formation, invasion abilities in vitro, and tumor growth in vivo. As expected, CCK-8 and colony formation assays demonstrated that miR-107 silencing could weaken the suppressive effects of LINC00152 
knockdown on cell proliferation and clone formation in U87 and U251 cells (Fig. 7a-b). Moreover, matrigel transwell assays showed that LINC00152 knockdown and inhibition of miR-107 caused opposite effects on the ability of GBM cell invasion. However, miR-107 inhibitor could partially reverse the effect of LINC00152 knockdown on cell invasion of GBM (Fig. 7c). Furthermore, by in vivo assays, tumor growth in xenografts with co-transfected sh-LINC00152 and miR-107-NC clones was decreased compared with that in negative control group, whereas miR-107 silencing eliminated the suppressor effect induced by LINC00152 knockdown on tumor growth (Fig. 7d-e). Collectively, these results suggest that the suppressive effects of LINC00152 knockdown on GBM could be reversed by miR-107 silencing both in vitro and in vivo.

\section{Discussion}

Glioblastoma multiforme (GBM) is the most prevalent and most lethal primary intrinsic brain tumor. It accounts for $50 \%$ of malignant glioma cases and is characterized histologically by considerable cellularity and mitotic activity, vascular proliferation, and necrosis(Omuro and DeAngelis 2013; Lathia et al. 2015). Although targeted therapies or immunotherapies have been used to treat GBM, maximal surgical resection followed by concurrent radiation therapy with temozolomide (TMZ) and subsequent additional adjuvant temozolomide (TMZ) therapy remains the standard therapy for GBM (Ricard et al. 2012;Omuro and DeAngelis 2013). Although accepted the standard therapy, GBM patient prognosis is still clinically frustrating (Wen and Kesari 2008;Stupp et al. 2009). To better understand and to find more effective treatments for this disease, it is vital to identify novel biomarkers and therapeutic targets. Combining bioinformatics analysis and biological experiments, our present study revealed that LINC00152 is a potential prognostic marker for glioma patients and play an oncogene role in GBM.

LINC00152 is one 828-bp IncRNA and locates at chromosome 2p11.2. Increasing researches indicate LINC00152 plays an oncogene role in many cancers and may act as a diagnostic and prognostic biomarker for them (Yu et al. 2017). For example, Wu et al (Wu et al. 2016) LINC00152 was significantly upregulated in clear cell renal cell carcinoma, may serve as an independent predictor of overall survival and can promote cell proliferation and invasion, inhibit cell cycle and apoptosis. In this study, we found that the expression of LINCO0152 is not only dramatically upregulated, also significantly associated with WHO grade, IDH status, MGMT promoter status and transcriptome subtype in glioma. The promoter methylation of MGMT is clinically used as a biomarker of response to alkylating agents for glioma (Esteller et al. 2000). Thus, LINC00152 may be related to the sensitivity of glioma to TMZ chemotherapy, which needs to be confirmed by further study. Furthermore, this study indicated that $\angle I N C 00152$ is an independent prognostic factor for glioma patients and that low levels of LINC00152 expression predict better prognosis. Functionally, through loss-of-function approaches, we fund that knocked down the expression of LINC00152 significantly inhibited cell proliferation, colony formation, invasion, and induced cell apoptosis in vitro and decreased tumor growth in vivo, which was consistent with previous studies(Cai et al. 2018;Liu et al. 2018). Taken together, LINC00152 is a potential prognostic marker and therapeutic target for glioma patient and acts as an oncogene in GBM. 
Certain IncRNAs have been proposed to function as "miRNA sponges"(Ebert and Sharp 2010;Beermann et al. 2016), they contain miRNA response elements (MREs) that can sequester miRNAs, thereby, preventing the miRNAs from binding to their target genes (Ebert and Sharp 2010;Beermann et al. 2016;Bhan et al. 2017). For example, IncRNA LINC00673, regulates non-small cell lung cancer proliferation, migration, invasion, and epithelial-mesenchymal transition by sequestering miR-150-5p(Lu et al. 2017). LncRNA SPRY4-IT1 acts as a sponge RNA to sequester miR-101-3p to promote proliferation and metastasis of bladder cancer cells through upregulation of EZH2(Liu et al. 2017). As a member of IncRNAs, LINC00152 is also reported that it can function as "miRNA sponges" to many miRNAs, including miR-497, miR-608, miR-153-3p, miR-138 and so on(Cai et al. 2017;Liu et al. 2019;Ouyang et al. 2019; Sun et al. 2019). It is not difficult to find that the one IncRNA can regulate multiple miRNAs. Here, we confirmed that LINC00152 acted as a miRNA sponge for $m i R-107$ and regulated the expression of miR-107. Chen et al reported that miR-107 is downregulated in glioma and overexpression of miR-107could inhibit proliferation of glioma(Chen et al. 2016). Besides, our results showed that LINC00152 plays oncogenic role depending miR-107 in vitro and in vivo.

$R A B 10$, a member of the Ras small GTPase superfamily, is a protein-coding gene with GTP and GDP binding domains(Just and Peranen 2016; Jaldin-Fincati et al. 2017). RAB10 participates in the insulinstimulated translocation of GLUT4 in adipocytes(Sano et al. 2011), basement membrane secretion(Lerner et al. 2013), and the formation and maintenance of the endoplasmic reticulum (English and Voeltz 2013). As a member of the RAS oncogene family, recent studies have showed that $R A B 10$ is closely related to tumorigenesis and cancer development. In hepatocellular carcinoma, RAB10 overexpression promotes tumor growth through multiple oncogenic pathways, cell stress, and apoptosis pathways and indicates a poor prognosis for HCC patients(Wang et al. 2017). Jiang et al identified RAB10 as a target of miR-329 and found that miR-329 was able to inhibit osteosarcoma cell proliferation, promote apoptosis, and induce G0/G1 cell cycle arrest via $R A B 10$ (Jiang et al. 2016). In this study, our study showed that $R A B 10$ was a target of miR-107 and further revealed the regulation mechanism among LINC00152, miR-107 and $R A B 10$. Additionally, we also fund that $R A B 10$ is upregulated in glioma, which suggests it may be an oncogene for glioma.

The mechanism of linc00152 in glioma is complex. Cai et al(Cai et al. 2018) found that LINC00152 acted as a miRNA sponge for miR-612 in GBM cells, negatively regulated miR-612 releases, which resulted in the elevated $A K T 2$, activated NF-KB pathway to promote proneural-mesenchymal transition(Cai et al. 2018). Liu et al reported that overexpression of LINC00152 suppressed miR-107 expression in U87 cells and enhanced the expression of HMGA2, a direct target gene of miR-107(Liu et al. 2018). In this study we revealed the new regulatory network of $L I N C 00152$, miR-107, and RAB10, which may enrich our understanding of the mechanism of LINCO0152 in glioma and provide novel strategy for the treatment of glioma.

\section{Conclusion}


LINC00152 is aberrantly upregulated in gliomas and may be a valuable prognostic marker for glioma patients. The LINC00152 plays a pivotal role in regulation of the glioma malignancy via LINC00152/MIR$107 / R A B 10$ axis. Therefore, targeting this axis might be an effective therapeutic strategy to treat glioma.

\section{Declarations}

\section{Acknowledgments}

The authors would like to thank the support of the National Nature Science Foundation of China (No 81801908 Gang Peng), the Science and Technology Planning Project of Guangzhou (No 201803010013, Songhua Xiao) and like to thank Andrew V. Yang for providing help with writing this manuscript.

\section{Funding}

This study was supported by grants from the National Nature Science Foundation of China (No 81801908) and the Science and Technology Planning Project of Guangzhou (No 201803010013).

\section{Availability of data and materials}

All data generated or analyzed during this study are included in this published article.

\section{Code availability}

Not applicable

\section{Authors' contributions}

GP mainly performed the experiments, analyzed the data and wrote the paper. JS performed construction of vectors and cells culture and transfection. ZW performed western blot and prepared the samples. QL and SX carried out the experiment design, manuscript drafting, and revision. All authors read and approved the final manuscript.

\section{Ethics approval and consent to participate}

Written informed consent was obtained from all participants included in this study.

\section{Patient consent for publication}

Written informed consent for publication was obtained from all participants.

\section{Competing interests}

The authors declare that they have no competing financial interests.

\section{References}


1. Beermann J, Piccoli MT, Viereck J, Thum T. Non-coding RNAs in Development and Disease: Background, Mechanisms, and Therapeutic Approaches. Physiol Rev. 2016;96:1297-325.

2. Bhan A, Soleimani M, Mandal SS. Long Noncoding RNA and Cancer: A New Paradigm. Cancer Res. 2017;77:3965-81.

3. Cai J, Zhang J, Wu P, Yang W, Ye Q, Chen Q, Jiang C. (2018). Blocking LINC00152 suppresses glioblastoma malignancy by impairing mesenchymal phenotype through the miR-612/AKT2/NFkappaB pathway. J Neurooncol.

4. Cai Q, Wang Z, Wang S, Weng M, Zhou D, Li C, Wang J, Chen E, Quan Z. (2017). Long non-coding RNA LINC00152 promotes gallbladder cancer metastasis and epithelial-mesenchymal transition by regulating HIF-1alpha via miR-138. Open Biol 7.

5. Chen L, Li ZY, Xu SY, Zhang XJ, Zhang Y, Luo K, Li WP. Upregulation of miR-107 Inhibits Glioma Angiogenesis and VEGF Expression. Cell Mol Neurobiol. 2016;36:113-20.

6. Chen QN, Chen X, Chen ZY, Nie FQ, Wei CC, Ma HW, Wan L, Yan S, Ren SN, Wang ZX. Long intergenic non-coding RNA 00152 promotes lung adenocarcinoma proliferation via interacting with EZH2 and repressing IL24 expression. Mol Cancer. 2017;16:17.

7. Deguchi S, Katsushima K, Hatanaka A, Shinjo K, Ohka F, Wakabayashi T, Zong H, Natsume A, Kondo Y. Oncogenic effects of evolutionarily conserved noncoding RNA ECONEXIN on gliomagenesis. Oncogene. 2017;36:4629-40.

8. Ebert MS, Sharp PA. Emerging roles for natural microRNA sponges. Curr Biol. 2010;20:R858-61.

9. English AR, Voeltz GK. Rab10 GTPase regulates ER dynamics and morphology. Nat Cell Biol. 2013;15:169-78.

10. Esteller M, Garcia-Foncillas J, Andion E, Goodman SN, Hidalgo OF, Vanaclocha V, Baylin SB, Herman JG. Inactivation of the DNA-repair gene MGMT and the clinical response of gliomas to alkylating agents. N Engl J Med. 2000;343:1350-4.

11. Fan C, Tang Y, Wang J, Xiong F, Guo C, Wang Y, Zhang S, Gong Z, Wei F, Yang L, He Y, Zhou M, Li X, Li $\mathrm{G}$, Xiong W, Zeng Z. Role of long non-coding RNAs in glucose metabolism in cancer. Mol Cancer. 2017; 16:130.

12. Fang $X Y$, Pan HF, Leng RX, Ye DQ. Long noncoding RNAs: novel insights into gastric cancer. Cancer Lett. 2015;356:357-66.

13. Feng S, Zhang J, Su W, Bai S, Xiao L, Chen X, Lin J, Reddy RM, Chang AC, Beer DG, Chen G. Overexpression of LINC00152 correlates with poor patient survival and knockdown impairs cell proliferation in lung cancer. Sci Rep. 2017;7:2982.

14. Floris G, Zhang L, Follesa P, Sun T. Regulatory Role of Circular RNAs and Neurological Disorders. Mol Neurobiol. 2017;54:5156-65.

15. Furnari FB, Cloughesy TF, Cavenee WK, Mischel PS. Heterogeneity of epidermal growth factor receptor signalling networks in glioblastoma. Nat Rev Cancer. 2015;15:302-10. 
16. Gomes CC, De Sousa SF, Calin GA, Gomez RS. The emerging role of long noncoding RNAs in oral cancer. Oral Surg Oral Med Oral Pathol Oral Radiol. 2017;123:235-41.

17. Gummalla M, Galetti S, Maeda RK, Karch F. Hox gene regulation in the central nervous system of Drosophila. Front Cell Neurosci. 2014;8:96.

18. Guo H, Hu G, Yang Q, Zhang P, Kuang W, Zhu X, Wu L. Knockdown of long non-coding RNA CCAT2 suppressed proliferation and migration of glioma cells. Oncotarget. 2016;7:81806-14.

19. Huarte M. The emerging role of IncRNAs in cancer. Nat Med. 2015;21:1253-61.

20. Jaldin-Fincati JR, Pavarotti M, Frendo-Cumbo S, Bilan PJ, Klip A. Update on GLUT4 Vesicle Traffic: A Cornerstone of Insulin Action. Trends Endocrinol Metab. 2017;28:597-611.

21. Ji J, Tang J, Deng L, Xie Y, Jiang R, Li G, Sun B. LINC00152 promotes proliferation in hepatocellular carcinoma by targeting EpCAM via the mTOR signaling pathway. Oncotarget. 2015;6:42813-24.

22. Jia P, Cai H, Liu X, Chen J, Ma J, Wang P, Liu Y, Zheng J, Xue Y. Long non-coding RNA H19 regulates glioma angiogenesis and the biological behavior of glioma-associated endothelial cells by inhibiting microRNA-29a. Cancer Lett. 2016;381:359-69.

23. Jiang W, Liu J, Xu T, Yu X. MiR-329 suppresses osteosarcoma development by downregulating Rab10. FEBS Lett. 2016;590:2973-81.

24. Just WW, Peranen J. Small GTPases in peroxisome dynamics. Biochim Biophys Acta. 2016;1863:1006-13.

25. Katsushima K, Natsume A, Ohka F, Shinjo K, Hatanaka A, Ichimura N, Sato S, Takahashi S, Kimura H, Totoki Y, Shibata T, Naito M, Kim HJ, Miyata K, Kataoka K, Kondo Y. Targeting the Notch-regulated non-coding RNA TUG1 for glioma treatment. Nat Commun. 2016;7:13616.

26. Lathia JD, Mack SC, Mulkearns-Hubert EE, Valentim CL, Rich JN. Cancer stem cells in glioblastoma. Genes Dev. 2015;29:1203-17.

27. Lerner DW, Mccoy D, Isabella AJ, Mahowald AP, Gerlach GF, Chaudhry TA, Horne-Badovinac S. A Rab10-dependent mechanism for polarized basement membrane secretion during organ morphogenesis. Dev Cell. 2013;24:159-68.

28. Liu D, Gao M, Wu K, Zhu D, Yang Y, Zhao S. LINC00152 facilitates tumorigenesis in esophageal squamous cell carcinoma via miR-153-3p/FYN axis. Biomed Pharmacother. 2019;112:108654.

29. Liu D, Li Y, Luo G, Xiao X, Tao D, Wu X, Wang M, Huang C, Wang L, Zeng F, Jiang G. LncRNA SPRY4IT1 sponges miR-101-3p to promote proliferation and metastasis of bladder cancer cells through upregulating EZH2. Cancer Lett. 2017;388:281-91.

30. Liu X, Yidayitula Y, Zhao H, Luo Y, Ma X, Xu M. LncRNA LINC00152 promoted glioblastoma progression through targeting the miR-107 expression. Environ Sci Pollut Res Int. 2018;25:17674-81.

31. Lu W, Zhang H, Niu Y, Wu Y, Sun W, Li H, Kong J, Ding K, Shen HM, Wu H, Xia D, Wu Y. Long noncoding RNA linc00673 regulated non-small cell lung cancer proliferation, migration, invasion and epithelial mesenchymal transition by sponging miR-150-5p. Mol Cancer. 2017;16:118. 
32. Omuro A, Deangelis LM. Glioblastoma and other malignant gliomas: a clinical review. JAMA. 2013;310:1842-50.

33. Ouyang T, Zhang Y, Tang S, Wang Y. Long non-coding RNA LINC00052 regulates miR-608/EGFR axis to promote progression of head and neck squamous cell carcinoma. Exp Mol Pathol. 2019;111:104321.

34. Pastori C, Kapranov P, Penas C, Peschansky V, Volmar CH, Sarkaria JN, Bregy A, Komotar R, St Laurent G, Ayad NG, Wahlestedt C. The Bromodomain protein BRD4 controls HOTAIR, a long noncoding RNA essential for glioblastoma proliferation. Proc Natl Acad Sci U S A. 2015;112:832631.

35. Peng G, Yuan X, Yuan J, Liu Q, Dai M, Shen C, Ma J, Liao Y, Jiang W. miR-25 promotes glioblastoma cell proliferation and invasion by directly targeting NEFL. Mol Cell Biochem. 2015;409:103-11.

36. Ricard D, Idbaih A, Ducray F, Lahutte M, Hoang-Xuan K, Delattre JY. Primary brain tumours in adults. Lancet. 2012;379:1984-96.

37. Sa L, Li Y, Zhao L, Liu Y, Wang P, Liu L, Li Z, Ma J, Cai H, Xue Y. The Role of HOTAIR/miR-148b3p/USF1 on Regulating the Permeability of BTB. Front Mol Neurosci. 2017;10:194.

38. Sano H, Peck GR, Kettenbach AN, Gerber SA, Lienhard GE. Insulin-stimulated GLUT4 protein translocation in adipocytes requires the Rab10 guanine nucleotide exchange factor Dennd4C. J Biol Chem. 2011;286:16541-5.

39. Siegel RL, Miller KD, Jemal A. Cancer Statistics, 2017. CA Cancer J Clin. 2017;67:7-30.

40. Stupp R, Hegi ME, Mason WP, Van Den Bent MJ, Taphoorn MJ, Janzer RC, Ludwin SK, Allgeier A, Fisher B, Belanger K, Hau P, Brandes AA, Gijtenbeek J, Marosi C, Vecht CJ, Mokhtari K, Wesseling P, Villa S, Eisenhauer E, Gorlia T, Weller M, Lacombe D, Cairncross JG, Mirimanoff RO, European Organisation For R, Treatment of Cancer Brain T, Radiation Oncology G, and National Cancer Institute of Canada Clinical Trials G (2009). Effects of radiotherapy with concomitant and adjuvant temozolomide versus radiotherapy alone on survival in glioblastoma in a randomised phase III study: 5-year analysis of the EORTC-NCIC trial. Lancet Oncol 10: 459-466.

41. Su J, Long W, Ma Q, Xiao K, Li Y, Xiao Q, Peng G, Yuan J, Liu Q. Identification of a Tumor Microenvironment-Related Eight-Gene Signature for Predicting Prognosis in Lower-Grade Gliomas. Front Genet. 2019;10:1143.

42. Su J, Ma Q, Long W, Tang H, Wu C, Luo M, Wang X, Xiao K, Li Y, Xiao Q, Zhang C, Li H, Liu Q. LCTL Is a Prognostic Biomarker and Correlates With Stromal and Immune Infiltration in Gliomas. Front Oncol. 2019;9:1083.

43. Sun Z, Guo X, Zang M, Wang P, Xue S, Chen G. Long non-coding RNA LINC00152 promotes cell growth and invasion of papillary thyroid carcinoma by regulating the miR-497/BDNF axis. J Cell Physiol. 2019;234:1336-45.

44. Teng W, Qiu C, He Z, Wang G, Xue Y, Hui X. Linc00152 suppresses apoptosis and promotes migration by sponging miR-4767 in vascular endothelial cells. Oncotarget. 2017;8:85014-23. 
45. Wang W, Jia WD, Hu B, Pan YY. RAB10 overexpression promotes tumor growth and indicates poor prognosis of hepatocellular carcinoma. Oncotarget. 2017;8:26434-47.

46. Wang Z, Guo Q, Wang R, Xu G, Li P, Sun Y, She X, Liu Q, Chen Q, Yu Z, Liu C, Xiong J, Li G, Wu M. The D Domain of LRRC4 anchors ERK1/2 in the cytoplasm and competitively inhibits MEK/ERK activation in glioma cells. J Hematol Oncol. 2016;9:130.

47. Wang Z, Yang J, Xu G, Wang W, Liu C, Yang H, Yu Z, Lei Q, Xiao L, Xiong J, Zeng L, Xiang J, Ma J, Li G, Wu M. Targeting miR-381-NEFL axis sensitizes glioblastoma cells to temozolomide by regulating stemness factors and multidrug resistance factors. Oncotarget. 2015;6:3147-64.

48. Wen PY, Kesari S. Malignant gliomas in adults. N Engl J Med. 2008;359:492-507.

49. Wu X, Wang Y, Yu T, Nie E, Hu Q, Wu W, Zhi T, Jiang K, Wang X, Lu X, Li H, Liu N, Zhang J, You Y. Blocking MIR155HG/miR-155 axis inhibits mesenchymal transition in glioma. Neuro Oncol. 2017;19:1195-205.

50. Wu Y, Tan C, Weng WW, Deng Y, Zhang QY, Yang XQ, Gan HL, Wang T, Zhang PP, Xu MD, Wang YQ, Wang CF. Long non-coding RNA Linc00152 is a positive prognostic factor for and demonstrates malignant biological behavior in clear cell renal cell carcinoma. Am J Cancer Res. 2016;6:285-99.

51. Yu H, Xue Y, Wang P, Liu X, Ma J, Zheng J, Li Z, Li Z, Cai H, Liu Y. Knockdown of long non-coding RNA $X I S T$ increases blood-tumor barrier permeability and inhibits glioma angiogenesis by targeting miR137. Oncogenesis. 2017;6:e303.

52. Yu M, Xue Y, Zheng J, Liu X, Yu H, Liu L, Li Z, Liu Y. Linc00152 promotes malignant progression of glioma stem cells by regulating miR-103a-3p/FEZF1/CDC25A pathway. Mol Cancer. 2017;16:110.

53. Yu Y, Yang J, Li Q, Xu B, Lian Y, Miao L. (2017). LINC00152: A pivotal oncogenic long non-coding RNA in human cancers. Cell Prolif 50.

54. Yuan J, Xiao G, Peng G, Liu D, Wang Z, Liao Y, Liu Q, Wu M, Yuan X. MiRNA-125a-5p inhibits glioblastoma cell proliferation and promotes cell differentiation by targeting TAZ. Biochem Biophys Res Commun. 2015;457:171-6.

55. Zhang JX, Han L, Bao ZS, Wang YY, Chen LY, Yan W, Yu SZ, Pu PY, Liu N, You YP, Jiang T, Kang CS, Glioma Cooperative C G. HOTAIR, a cell cycle-associated long noncoding RNA and a strong predictor of survival, is preferentially expressed in classical and mesenchymal glioma. Neuro Oncol. 2013;15:1595-603.

56. Zhu Y, Zhang X, Qi L, Cai Y, Yang P, Xuan G, Jiang Y. HULC long noncoding RNA silencing suppresses angiogenesis by regulating ESM-1 via the PI3K/Akt/mTOR signaling pathway in human gliomas. Oncotarget. 2016;7:14429-40.

\section{Tables}

Table 1: Univariate and multivariable Cox regression analysis of overall survival based on TCGA dataset 


\begin{tabular}{|c|c|c|c|c|}
\hline & Univariate analysis & & Multivariate analysis & \\
\hline & $\begin{array}{l}\text { HR } \\
(95 \% \text { Cl for HR) }\end{array}$ & $P$ & $\begin{array}{l}\text { HR } \\
(95 \% \text { Cl for HR) }\end{array}$ & $\mathrm{P}$ \\
\hline LINC00152 & $1.645(1.551-1.745)$ & $\star \star \star \star ~$ & $\begin{array}{l}1.206 \\
(1.081-1.345)\end{array}$ & $\star \star \star$ \\
\hline $\begin{array}{l}\text { gender } \\
\text { male VS Female }\end{array}$ & $1.225(0.955-1.572)$ & 0.110 & - & - \\
\hline $\begin{array}{l}\text { Age } \\
\leq 40 \text { VS }>40\end{array}$ & $0.231(0.165-0.324)$ & $\star \star \star$ & $0.3947(0.2565-0.6074)$ & $\star \star \star$ \\
\hline $\begin{array}{l}\text { grade } \\
\text { WHO II VS WHO III + IV }\end{array}$ & $0.178(0.121-0.262)$ & $\star \star \star *$ & $0.4497(0.2768-0.7306)$ & $\star \star$ \\
\hline $\begin{array}{l}\text { IDH status } \\
\text { WT VS MT }\end{array}$ & $\begin{array}{l}10.050 \\
(7.524-13.43)\end{array}$ & $\star \star \star \star ~$ & $3.205(1.863-5.514)$ & $\star \star *$ \\
\hline $\begin{array}{l}\text { MGMT promoter status } \\
\text { Methylation VS Unmethylation }\end{array}$ & $3.228(2.447-4.259)$ & $\star \star \star \star ~$ & - & - \\
\hline $\begin{array}{l}\text { Transcriptome subtype } \\
\text { NE + PN VS CL+ME }\end{array}$ & $0.152(0.113-0.203)$ & $\star \star \star *$ & $1.012(0.6335-1.618)$ & 0.959 \\
\hline
\end{tabular}

Abbreviations: $\mathrm{HR}$, hazard ratio, $\mathrm{Cl}$, Confidence interval.

\section{Figures}




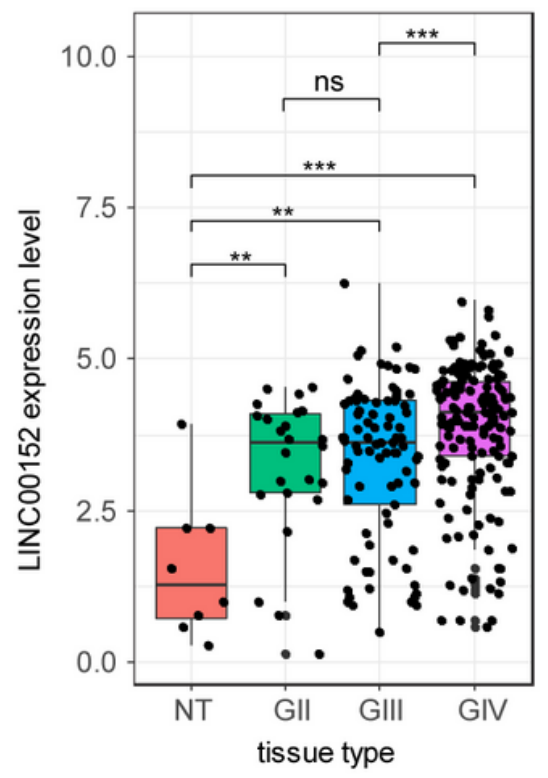

d

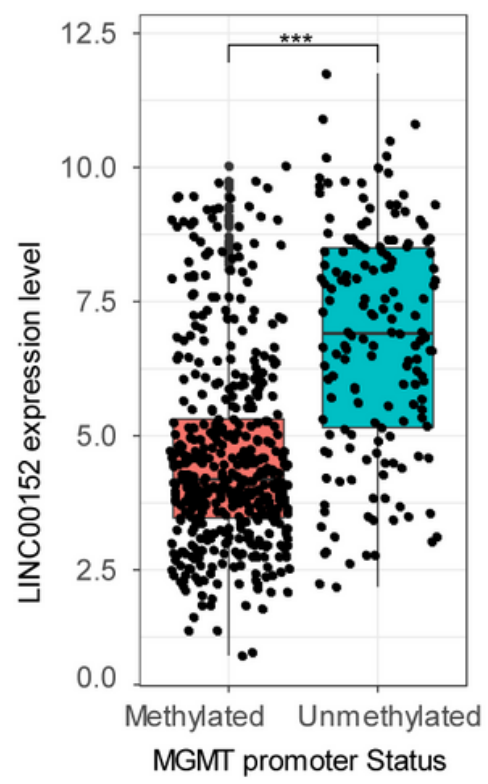

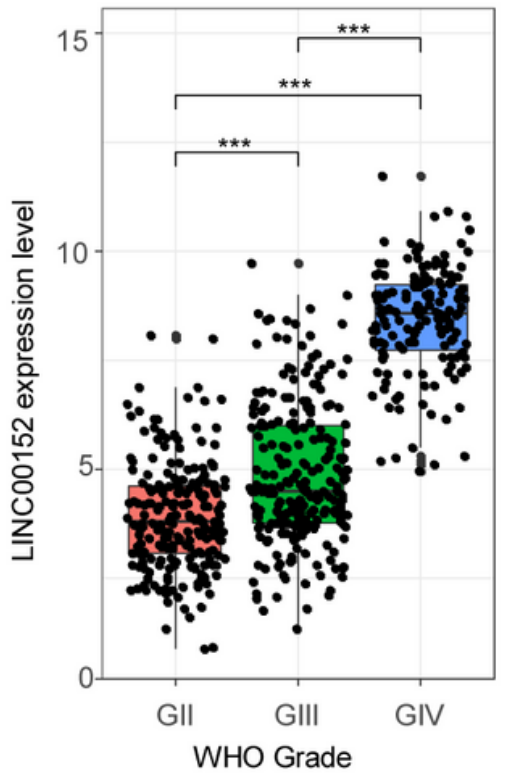

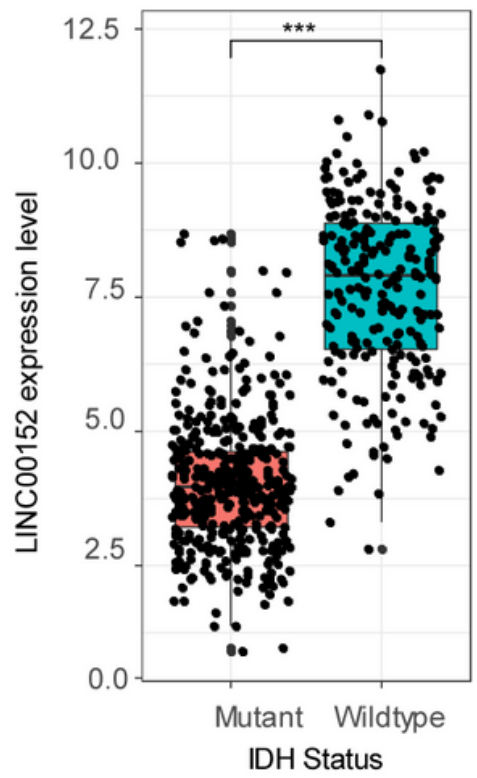

e

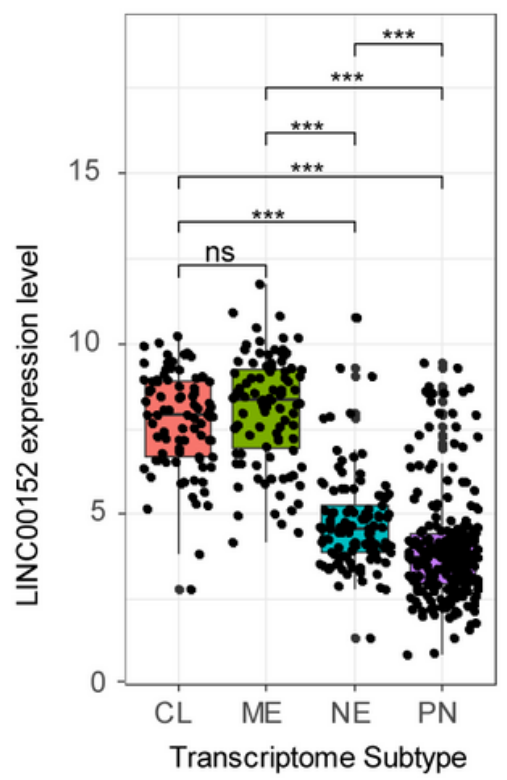

Figure 1

LINC00152 was significantly upregulated in gliomas and a prognostic factor for glioma patients a. The expression of LINC00152 between glioma tissues and normal brain tissues based on GSE16011 dataset. b. LINC00152 expression in glioma of WHO grade $\mathbb{X}-\mathbb{Z}$ based on the TCGA dataset. c. The expression of LINC00152 is significantly higher in IDH-Wildtype gliomas than that in IDH-Mutant disease based on the TCGA dataset. $d$. The expression of LINC00152 is significantly higher in gliomas with MGMT promoter unmethylation than that in gliomas with MGMT promoter methylation based on the TCGA dataset. e. LINC00152 expression pattern in different molecular subtypes of glioma (CL, ME, NE, PN) in the TCGA dataset. f. Kaplan-Meier curves for overall survival of patients with glioma with respect to levels of 
LINC00152 expression, which showed that high LINC00152 expression predicts poor prognosis for glioma patients based on the TCGA dataset. CL: classical, ME: mesenchymal, NE: neural, PN: proneural, NT: normal brain tissue, GII: grade II, GIII: grade III, GIV: grade IV, * $P<0.05$; ** $P<0.01 ; * \star * ~ P<0.001$

a

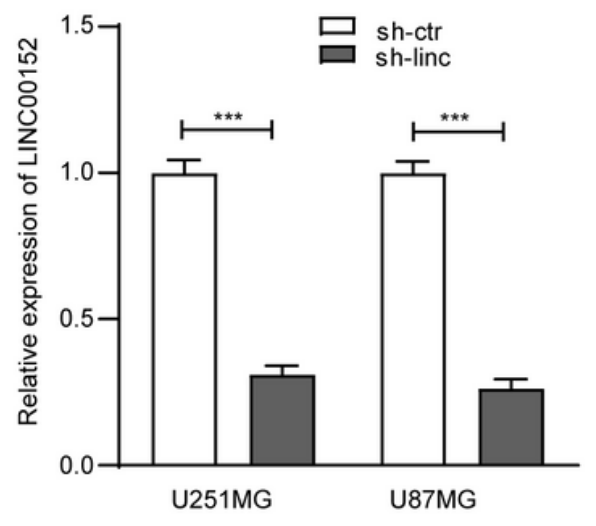

b

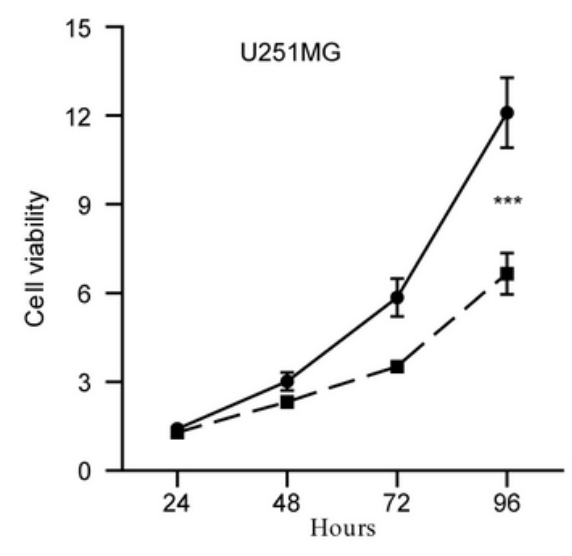

d

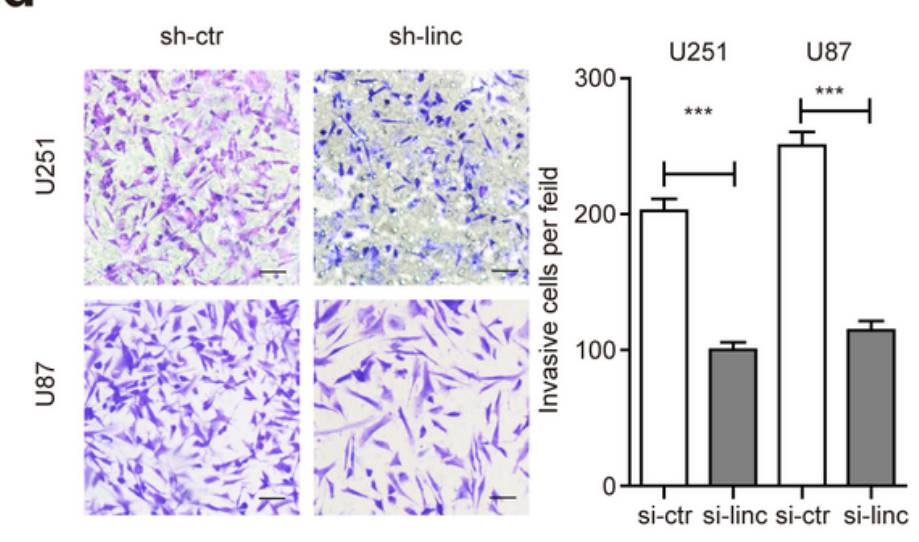

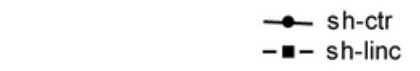

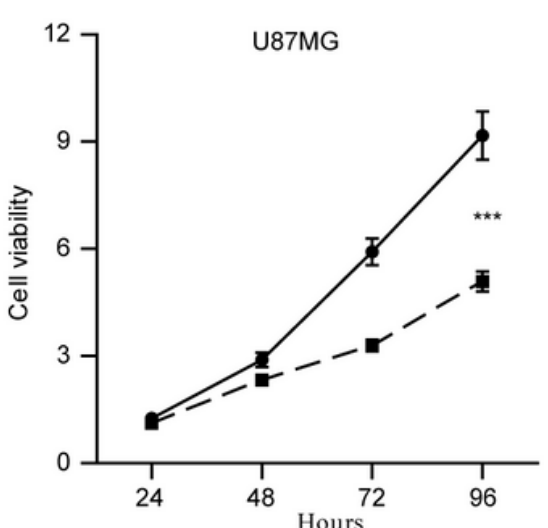

e

C
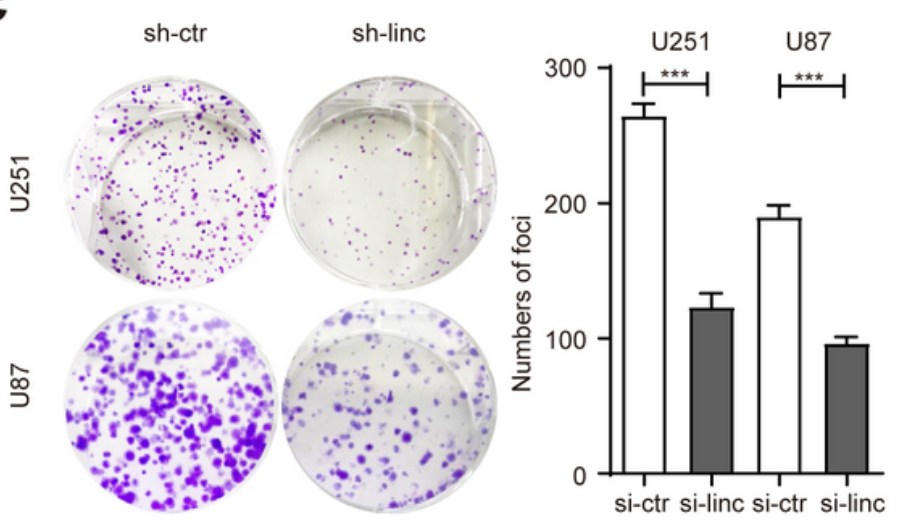
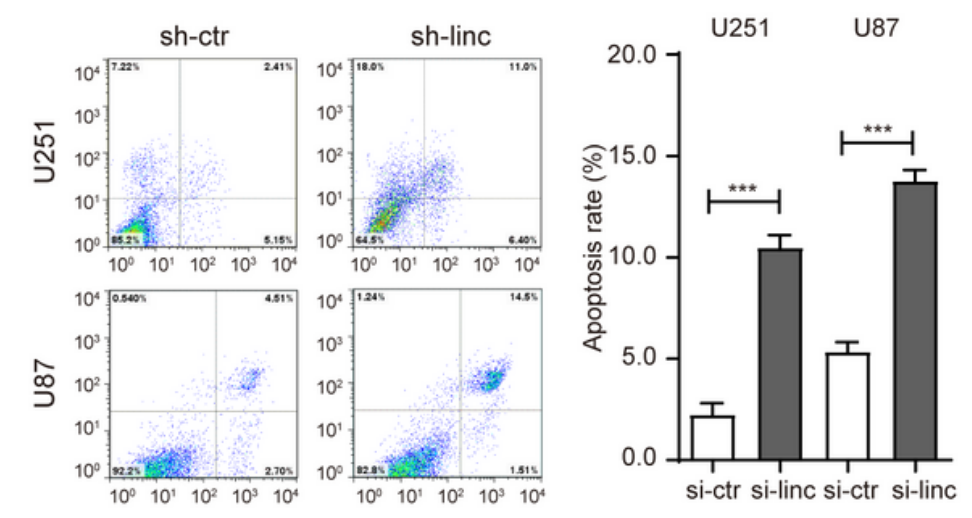

Figure 2

Knockdown of LINC00152 significantly inhibited proliferation, migration and invasion and induces apoptosis in vitro. a. Knockdown of LINC00152 expression using shRNA. shRNA dramatically suppressed LINC00152 expression at the RNA level compared with control shRNA (sh-ctr) in U251 and U87 cell lines, as determined by qRT-PCR. b. CCK-8 show reduced proliferation of U251 and U87 cells transfected with 
shRNA LINC00152 (sh-linc) compared with sh-ctr. c. Knockdown of LINC00152 inhibited colony formation ability in vitro. d. Matrigel chamber invasion assay showed reduced invasion of U251 and U87 cells after transfection with shRNA LINC00152 compared with control shRNA. e. Flow cytometry analysis of apoptosis showed that the knockdown of LINC00152 in U251 and U87 cells increased the percentage of apoptotic cells compared with sh-ctr. * $P<0.05$; ** $P<0.01$; *** $P<0.001$

a

\section{b}

sh-ctr

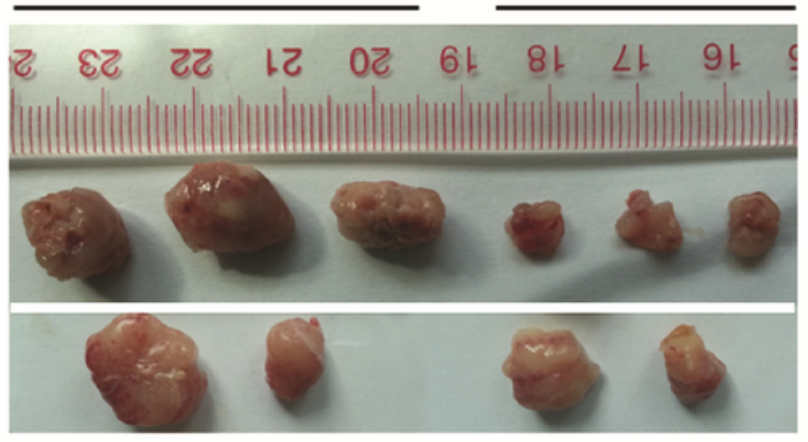

C

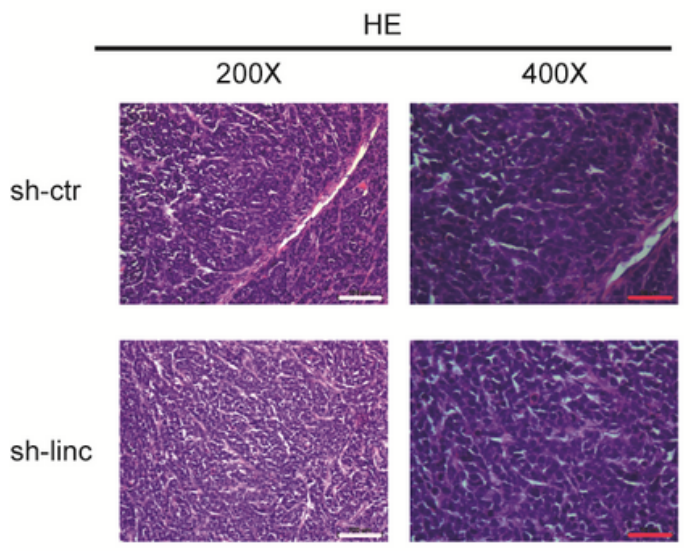

sh-linc sh-linc

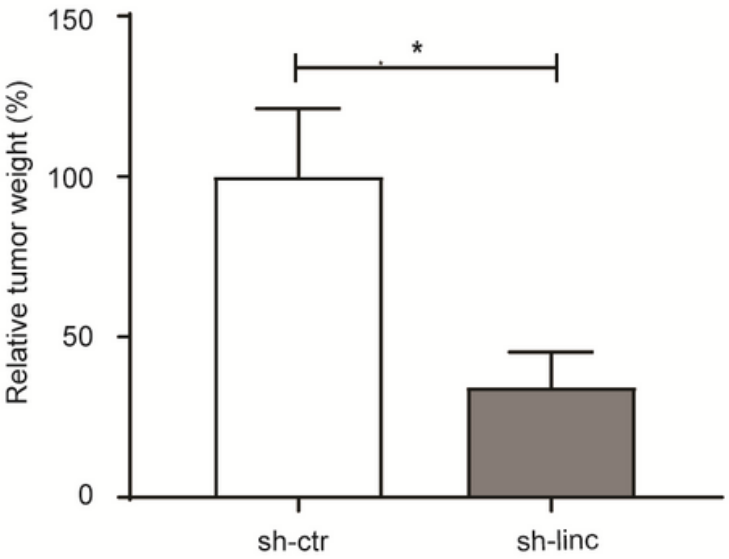

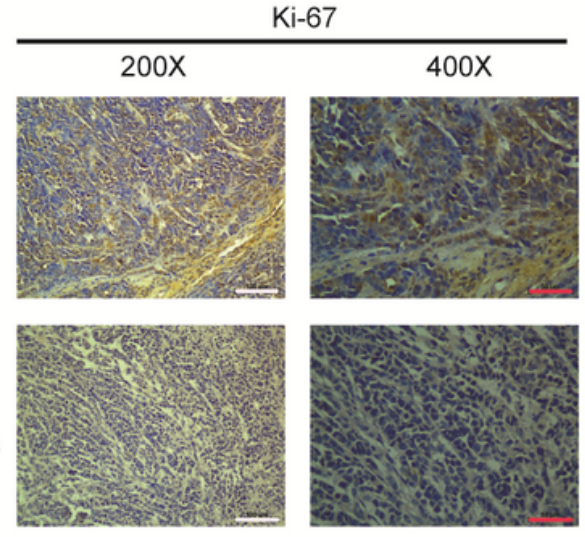

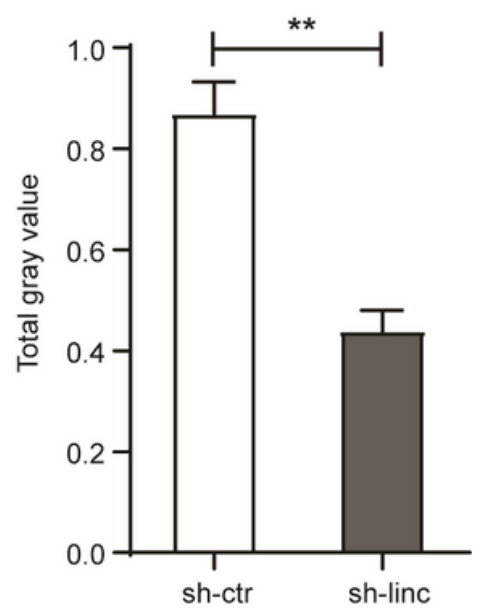

Figure 3

Knockdown of LINC00152 gene suppressed tumor growth in vivo. a. A representative picture of tumor xenograft morphology $35 \mathrm{~d}$ after injection. b. Tumor weights were measured after dissection from euthanized mice. c. H\&E staining of xenograft tumors isolated from mice that had been injected with U251 cells transfected with sh-ctr or sh-linc ( $\times 200$ magnification). Metastasis nodules are indicated by arrows. d. Representative images of Ki-67 immunohistochemistry from xenograft tumors $(\times 200$ magnification). ${ }^{\star} P<0.05 ;{ }^{* \star} \mathrm{P}<0.01$; ${ }^{* \star *} \mathrm{P}<0.001$; value of red bar and white bar were $50 \mu \mathrm{m}$ and $100 \mu \mathrm{m}$ respectively. 


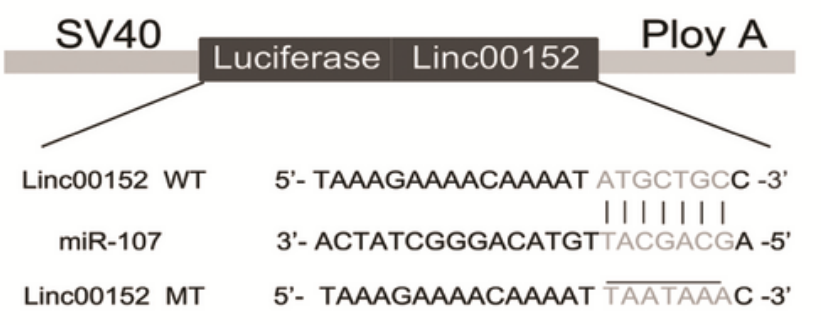

C

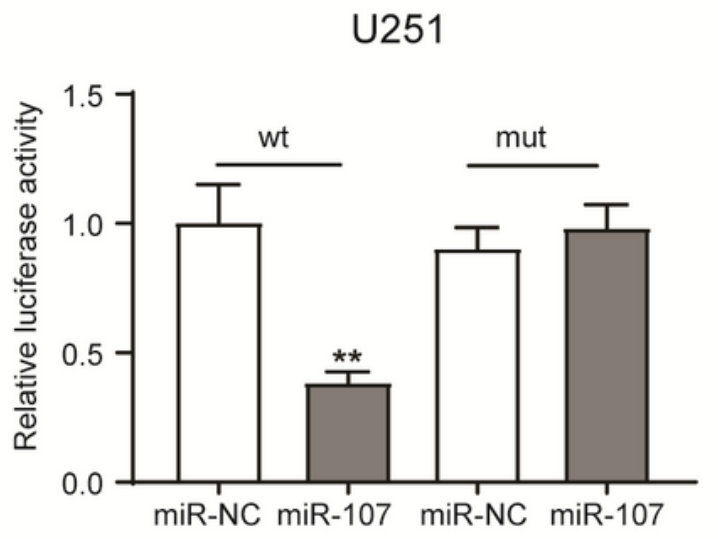

d

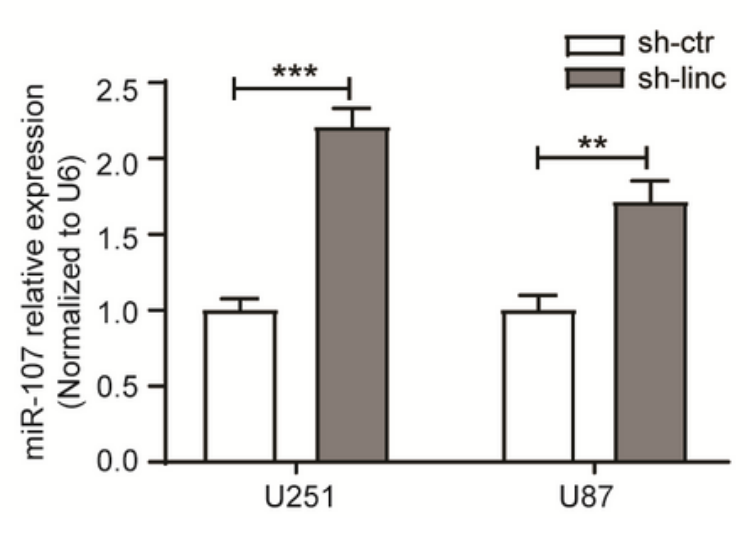

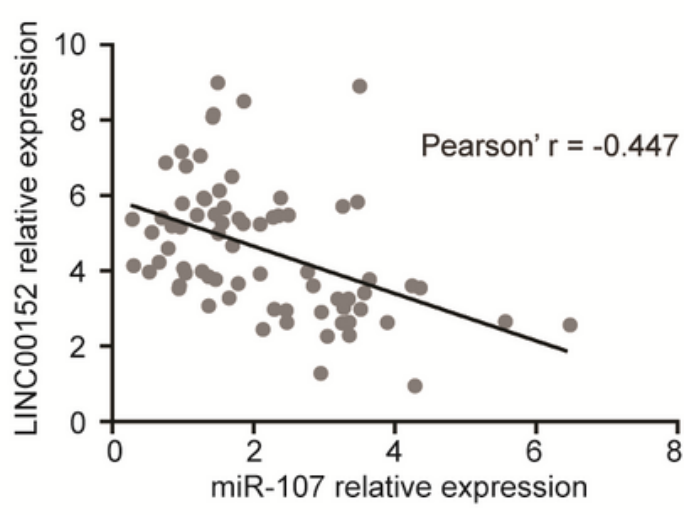

U87

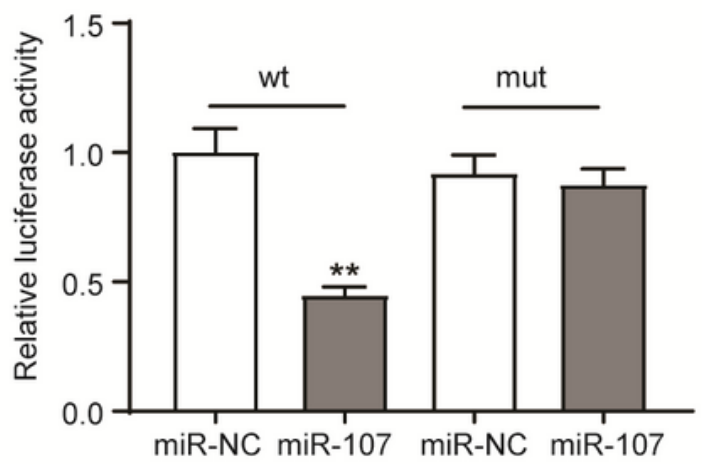

\section{Figure 4}

LINC00152 was a direct target of miR-107. a. Depiction of putative miR-107 binding sites on LINC00152 sequence. LINC00152 cDNA containing the putative miRNA recognition sites was cloned downstream of luciferase gene. b. Correlation analysis of the expression of LINC00152 and MIR-107 in glioma tissues. C. Luciferase assays of U251 and U87 cells co-transfected with either pMIR-REPORT-WT/MT LINC00152 
and miR-107 or negative control as indicated. d. mRNA levels of miR-107 in U251 and U87 cells transfected with LINC00152 shRNA or control shRNA. * $P<0.05$; ** $P<0.01$; *** $P<0.001$.

a

b
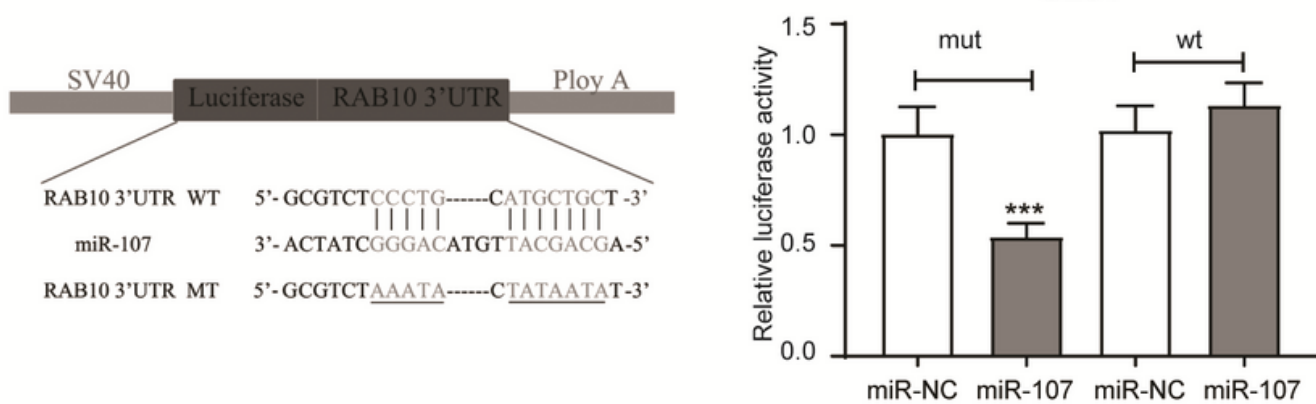

U251

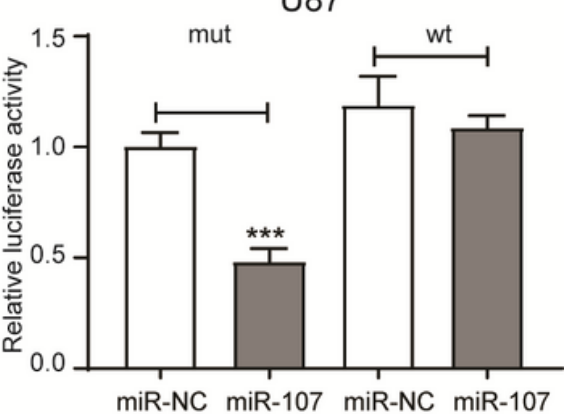

C
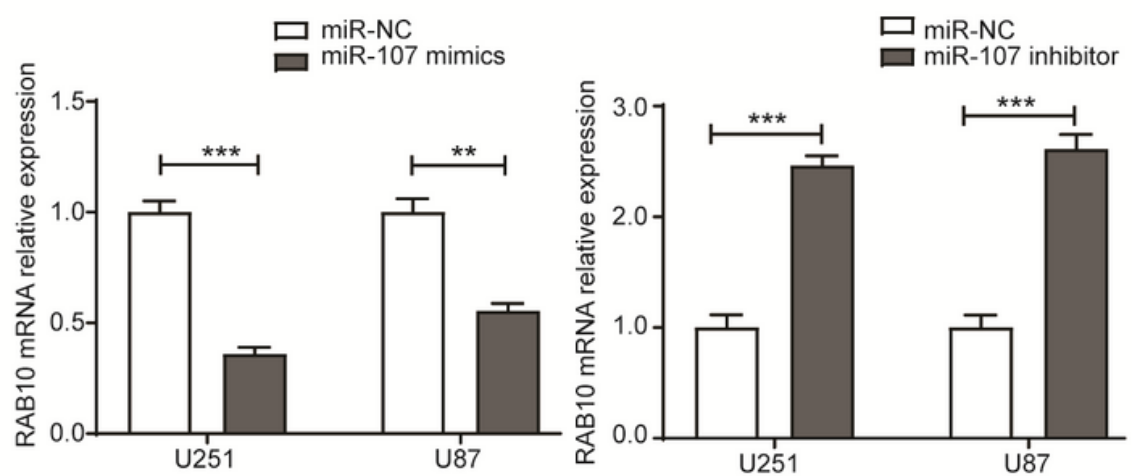

d

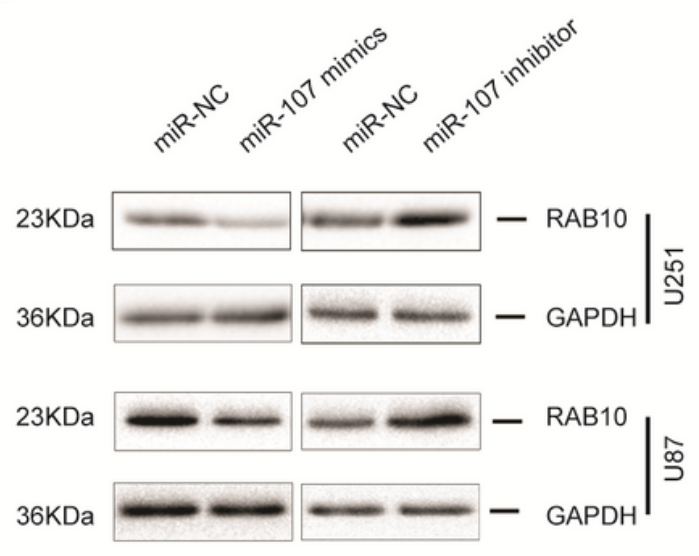

\section{Figure 5}

RAB10 was a direct target of miR-107. a. Depiction of miR-107 interaction sites in the 3'-UTR of RAB10. The 3'-UTR of a RAB10 cDNA containing miR-107 putative recognition sites was cloned 3' downstream of firefly luciferase gene. b. Luciferase assays of U251 and U87 cells co-transfected with either pMIRREPORT-WT/MT MALAT1 and miR-107 or negative controls as indicated. c. qRT-PCR showing mRNA levels of RAB10 $24 \mathrm{~h}$ after miR-107 mimics were transfected into U251 and U87 cells. d. Western blot analysis showing protein levels of RAB10 $24 \mathrm{~h}$ after miR-107 mimics or inhibitor were transfected into glioma cell lines. * $\mathrm{P}<0.05 ;$ ** $\mathrm{P}<0.01 ;$ *** $\mathrm{P}<0.001$ 

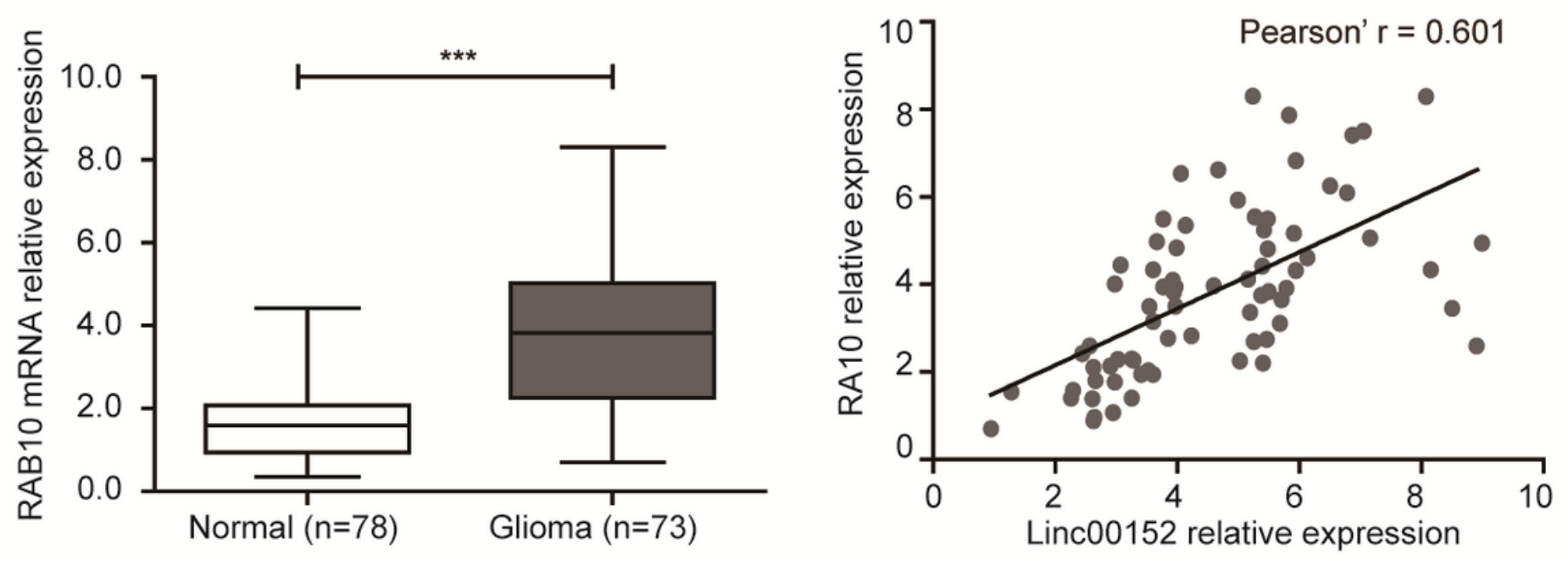

C
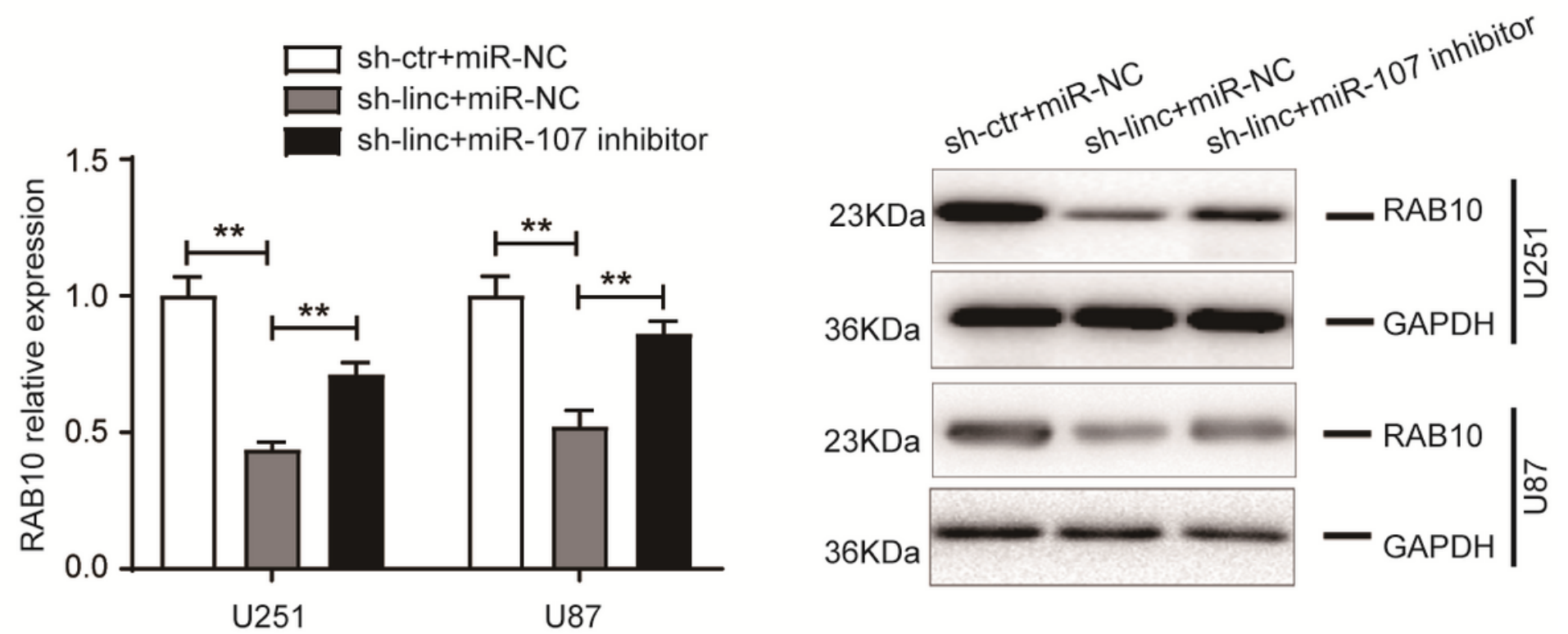

Figure 6

LINC00152 regulated expression of RAB10 gene through miR-107. a. Relative expression of RAB10 in glioma tissues ( $n=73$ ) compared with that in normal brain tissues $(n=78)$. RAB10 expression is determined using qRT-PCR and normalized to GAPDH expression levels. b. Correlation analysis of the expression of LINC00152 and RAB10 in glioma tissues. C. qRT-PCR showing mRNA levels of RAB10. Down-regulation of LINC00152 expression in glioma cell lines decreased mRNA levels of RAB10 and miR107 inhibitor reversed this effect. d. Western blot analysis showing RAB10 protein levels. The addition of miR-107 inhibitor reversed the effects of LINC00152 shRNA in U251 and U87 cells. ${ }^{*}<0.05$; $* \star P<0.01$; $\star \star \star P<0.001$ 

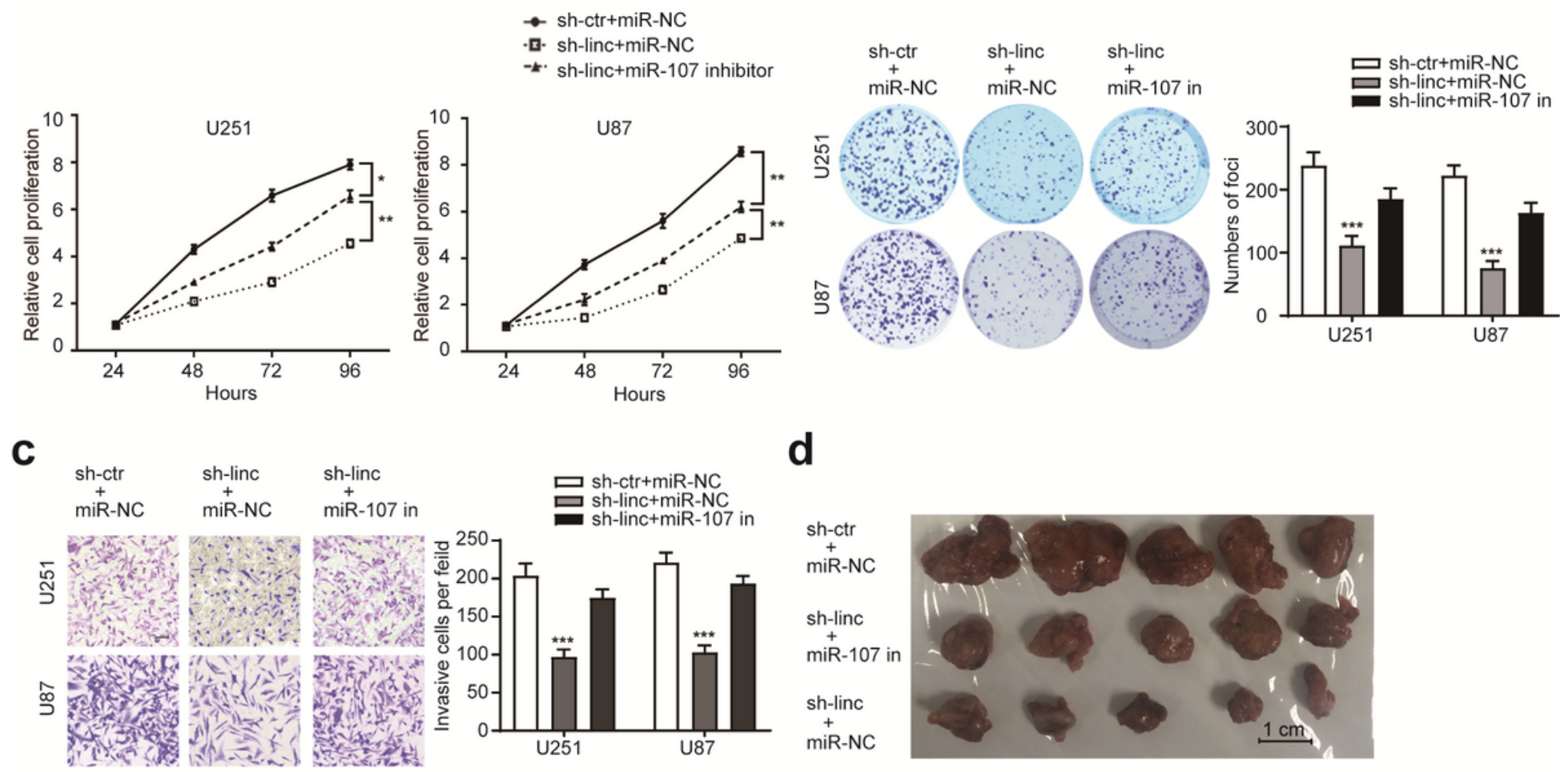

d

\section{e}

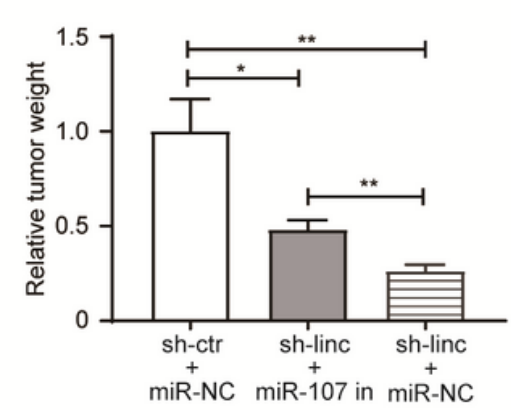

Figure 7

The effects induced by LINC00152 knockdown were reversed by miR-107 inhibition. a. CCK-8 assay showed that down-regulation of miR-107 partially reversed the inhibitory effect of proliferation induced by LINC00152 shRNA on U251 and U87 cell lines. b. Colony formation assay showed that down-regulation of miR-107 reversed the effects of LINC00152 shRNA on U251 and U87 cell lines. The miR-107 inhibitor increased the colony formation of U251 and U87 cells and reversed the effect of LINC00152 shRNA. C. Matrigel chamber invasion and migration assay showing reduced invasion of U251 and U87 cell lines after transfection with LINC00152 shRNA. The miR-107 inhibitor increased the invasion of U251 and U87 cells and reversed the effect of LINC00152 shRNA. d. A representative picture of tumor xenograft morphology $35 \mathrm{~d}$ after injection. e. Tumor weights were measured after dissection from euthanized mice. * $\mathrm{P}<0.05 ; * \star \mathrm{P}<0.01 ;$;** $\mathrm{P}<0.001$

\section{Supplementary Files}


This is a list of supplementary files associated with this preprint. Click to download.

- OnlineSupplementaryFig1.png 